\title{
Cusp Flail Severity
}

National Cancer Institute

\section{Source}

National Cancer Institute. Cusp Flail Severity. NCI Thesaurus. Code C127551.

The qualitative measurement of the severity of a flailing cardiac valve cusp. 